Textures and Microstructures, 1989, Vol. 10, pp. 117-134

Reprints available directly from the publisher

Photocopying permitted by license only

(C) 1989 Gordon and Breach Science Publishers Inc.

Printed in the United Kingdom

\title{
An Investigation into the Accuracy of the Vector Method by Comparison with ECP Measurements
}

\author{
R. SHIMIZU, J. HARASE and K. OHTA \\ R\&D Laboratories III, Nippon Steel Corporation, Edamitu, Yawata-Higashiku, \\ Kitakyusyu 805, Japan
}

(Received 20 June 1988; in final form 6 August 1988)

In an attempt to investigate the accuracy of the vector method for crystal texture analysis, a comparison has been made between the inversion result of the pole figure made by X-ray studies using the VM and the inversion result of the pole figure made by ECP. A comparison has been made between the inversion by the pole figure generated by direct measurement of orientations by ECP and the actual orientation distribution (measured by ECP) displayed in the same mode. The materials studied were recrystallized $\mathrm{Fe}-3 \% \mathrm{Si}$ and $\mathrm{Fe}-50 \% \mathrm{Ni}$. The main findings were:

- In the mean intensities of each individual Box, the inversion results of pole figures made from orientations determined by ECP were in good agreement with the inversion from (100) pole figures made by X-ray or actual orientation distribution (made by ECP) displayed in the same mode as the vector method.

- For $\mathrm{Fe}-3 \% \mathrm{Si}$, quite a good agreement was obtained between the results inverted from X-ray pole figure and the direce en asurement by ECP for the intensity distribution of minor texture component along $\zeta$ angle. It was concluded from these investigations that the inversion of the pole figure by the vector method is accurate enough for most practical purposes.

KEY WORDS: ODF analysis, Vector method, Individual orientation measurement, Electron diffraction, $\mathrm{Fe}-3 \% \mathrm{Si}, \mathrm{Fe}-50 \% \mathrm{Ni}$. 


\section{INTRODUCTION}

In order to compare the vector method (VM) and the harmonic method (HM) for texture analysis, experimental pole figures made by X-ray measurement or model textures are commonly utilized (Kitagawa et al., 1982; Pospiech et al., 1984; Matsuo et al., 1985, Esling et al., 1987).

When experimental pole figures are used, the accuracy and completeness of the $\mathrm{X}$-ray measurement is of extreme importance (Schaben, 1984). The method of making complete pole figures by combining the reflection and transmission pole figures is one of the most important factors in achieving high accuracy in the inversion of pole figures by the VM (Shimizu et al., 1984). When model pole figures are used, the method of generating a suitable intensity distribution for the model orientation is also an important factor in the accuracy of the inversion of pole figures (Matsuo et al., 1985). A Gaussian distribution is usually used. When experimental pole figures are utilized for the inversion either by VM or HM, it is rather difficult to say which method results in the greater accuracy as the "true texture" contained in the pole figure is not known.

If the "true texture" of the specimen can be determined by means other than X-ray measurements, then the real accuracy of the VM can be obtained by comparing the results of the VM analysis with this "true texture." Recently it has become possible to analyze orientations of grains larger than $5 \mu \mathrm{m}$ in diameter very accurately by the SEM-ECC-ECP technique. Computer processing allows rapid analysis (Shimizu et al., 1984). It is now possible to determine the accurate texture distribution in a specimen by analyzing orientations and sizes of several hundreds of grains by ECP without utilizing X-ray measurements. The texture analyzed by ECP doesn't include "ghost." In connection with this, the present authors have tried to evaluate the VM using texture obtained by ECP as the "true texture." There is, however, some limitation to the accuracy of the "true texture" compared with the texture obtained by the $\mathrm{X}$-ray diffraction method, because it is impossible to measure all the orientations of grains by ECP over the same area measured by the $X$-ray diffraction method. The definitions of the terms used in the present paper is summarized in Table 1 . The pole figures made from ECP measurement and X-ray measurement are called PFE and 
Table 1 Definition of symbols utilized in this paper

\begin{tabular}{|c|c|c|}
\hline Term & Meaning & Preparation method \\
\hline ODE & $\begin{array}{l}\text { Three dimensional orientation } \\
\text { distribution directly } \\
\text { obtained from ECP } \\
\text { measurements }\end{array}$ & $\begin{array}{l}\text { The angles } \psi \omega \zeta \text { were calculated from } \\
\text { (HKL) }\langle\text { UVW } \text { of each grain } \\
\text { measured by ECP. } \\
\text { Orientation distribution is then shown } \\
\text { in the same mode as the vector } \\
\text { method taking account of the area of } \\
\text { the each grain measured by ECP. }\end{array}$ \\
\hline PFX & $\begin{array}{l}\text { Pole figure made by X-ray } \\
\text { diffraction }\end{array}$ & \\
\hline PFE & $\begin{array}{l}\text { Pole figure made from } \\
\text { orientations measured by } \\
\text { ECP }\end{array}$ & $\begin{array}{l}\text { The angles }\left(\alpha_{i}, \beta_{i}\right)(i=1,2,3) \text { of }(100) \\
\text { poles for each grain were calculated } \\
\text { from }(\mathrm{HKL})\langle\mathrm{UVW}\rangle \text { measured by } \\
\text { ECP. } \\
\text { Then pole figure is made taking } \\
\text { account of the area of each grain } \\
\text { measured by ECP. }\end{array}$ \\
\hline ODVX & $\begin{array}{l}\text { Inversion result of PFX by } \\
\text { vector method }\end{array}$ & Inversion of PFX by vector method. \\
\hline ODVE & $\begin{array}{l}\text { Inversion result of PFE by } \\
\text { vector method }\end{array}$ & Inversion of PFE by vector method. \\
\hline
\end{tabular}

PFX respectively. The inversions of pole figures of PFE and PFX by VM are called ODVE and ODVX, respectively. The "true texture" measured by ECP and displayed in the same format as VM is called ODE which is represented as a function of $\Psi, \omega$ and $\zeta$, which are directly obtained from orientations of individual grains and their areas by ECP. It is now possible to evaluate the accuracy of VM by comparing these three-dimensional orientational distributions, ODE, ODVE and ODVX, respectively. The terms $\psi, \omega$ and $\zeta$ are the same as those defined in the VM.

Comparison of ODE and ODVX was made by utilizing a $\mathrm{Fe}-3 \% \mathrm{Si}$ specimen. As for the comparison of the ODE and ODVE, $\mathrm{Fe}-3 \% \mathrm{Si}$ and $\mathrm{Fe}-50 \% \mathrm{Ni}$ specimens were used. In order to detemine the accuracy of the inversion of the minor textural component by the VM, the results inverted by VM and those obtained by ECP were compared by considering the intensity distribution of the Goss orientation $(\{110\}\langle 001\rangle)$ rotated around the ND axis in a primary recrystallized $\mathrm{Fe}-3 \% \mathrm{Si}$. 


\section{Comparison of ODE and ODVX in $\mathrm{Fe}-3 \% \mathrm{Si}$ alloy}

The material used was cold rolled and recrystallized $\mathrm{Fe}-3 \% \mathrm{Si}$ sheet with a grain size distribution of between 30 and $300 \mu \mathrm{m}$ as shown in Figure 4(c). The measurement area of the pole figure determined by the X-ray diffraction method (PFX) is $15 \mathrm{~mm} \Phi$ as shown in Figure 1. The area selected for ECP measurement was made up of nine blocks of the size about $5 \mathrm{~mm} \times 5 \mathrm{~mm}$ to evenly cover the area measured by X-ray. From each block, the orientations of the 100 or so grains present were measured by ECP. The total number of grain orientations measured was 967 , which corresponds to $7.1 \%$ of the area measured by the X-ray diffraction method. The pole figure obtained by X-ray measurement (PFX) of the area shown in Figure 1 is given in Figure 2. The inversion result (ODVX) of this pole figure by the VM is shown in Figure 3.

The (HKL)[UVW] of individual grains are obtained by ECP. These are shown in the form of the (100) pole figure and the inverse pole figures in Figure 4(a) and (b), respectively. The areas of the grains are not considered in these figures. When normalized, $(\mathrm{HKL})[\mathrm{UVW}]$ are expressed as $\left(h_{0} k_{0} l_{0}\right)\left[u_{0} v_{0} w_{0}\right], \psi, \omega$ and $\zeta$ are obtained as follows; $\psi=\tan ^{-1}\left(-h_{0} / k_{0}\right) \omega=\tan ^{-1}\left(-k_{0} / l_{0}\right) \quad \xi=$ $\sin ^{-1}\left(-v_{0}+k_{0} w_{0}\left(1+l_{0}\right)^{-1}\right)$. The Box number of the individual grain, defined by the VM, is determined from $\psi$ and $\omega$. Based on the Box number, $\zeta$ and the area of the individual grains, ODE expressed in

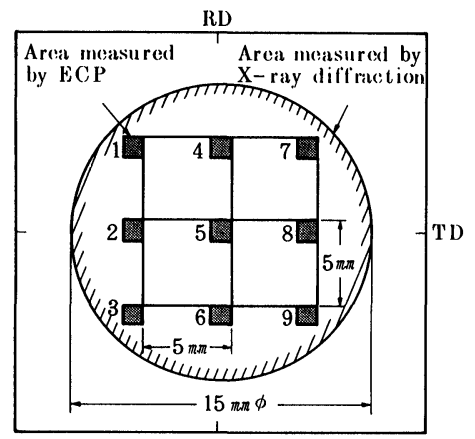

Figure 1 Schematic illustration of the area measured by ECP and X-ray diffraction method. Numbers in the figure denote the areas in which grain orientations were measured by ECP. 


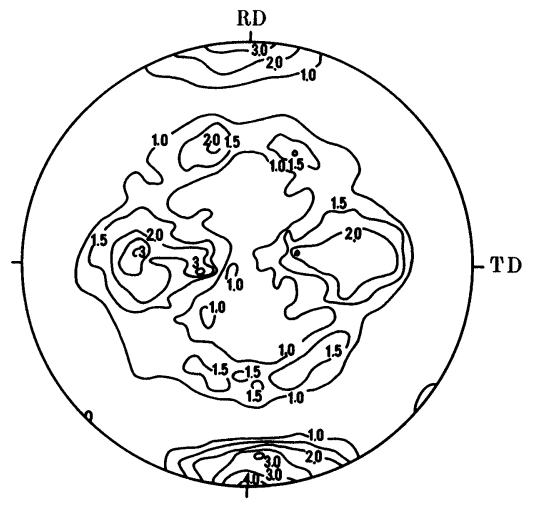

Figure 2 (100) pole figure (PFX) made by X-ray measurement of recrystallized $\mathrm{Fe}-3 \% \mathrm{Si}$.

exactly the same format as the VM is obtained. ODE is regarded as nearly representing "true texture" in the present paper. ODE based on the measurements of 967 grains are shown in Figure 5 in the symmetrical mode. The pole figure (PFE) made from the (HKL)[UVW] of individual grains and their areas is shown in Figure 6. PFE (Figure 6) and PFX (Figure 2) are in good qualitative

Box No.

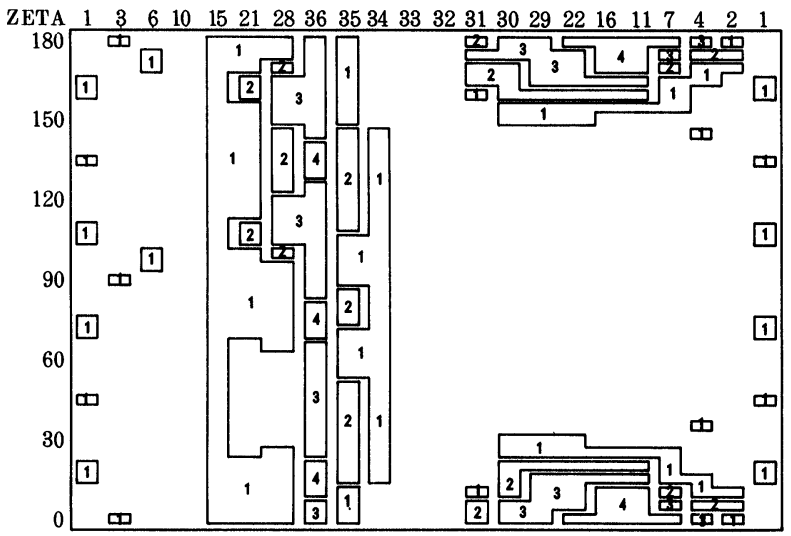

Figure 3 Three dimensional texture representation (ODVX) inverted by VM from the pole figure (Figure 2). 


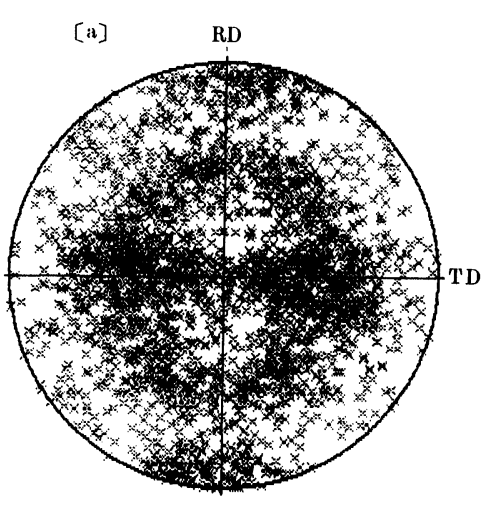

[b]

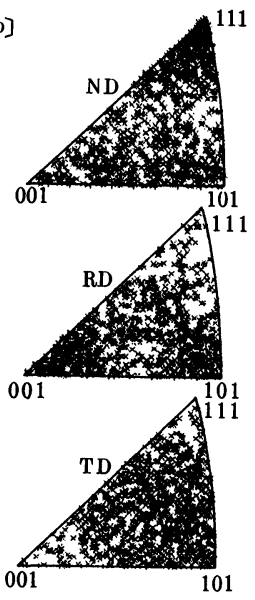

[C]

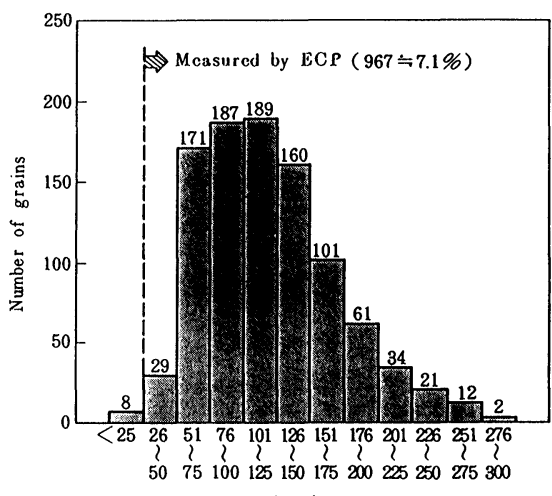

grain diameter $/ \mu m$

Figure 4 Orientation distribution and grain size distribution of 967 grains analyzed by ECP and ECC. (a) (100) pole figure, (b) Standard stereographic triangle projections (inverse pole figures), (c) Grain size distribution. The area of the grains are not taken into account in the (100) pole figure and the inverse pole figure.

agreement. This suggests that the texture measured by ECP is a good representation of the "true texture" in the area measured by the X-ray diffraction method. A comparison of the mean intensities of the individual Box numbers obtained by ODE and ODVX is shown in Figure 7. Fairly good agreement is obtained. This result indicates that the accuracy of the VM in generating the inverse pole figure is good. 
Box No.

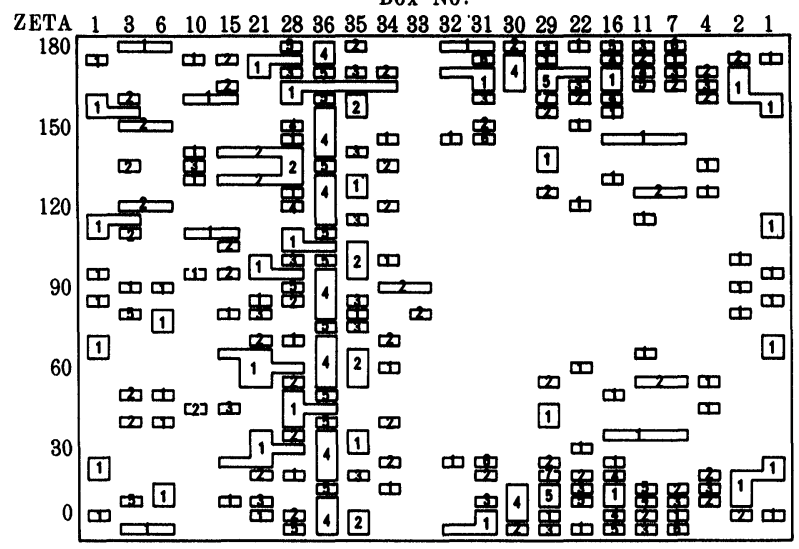

Figure 5 Three dimensional texture (ODE) of $\mathrm{Fe}-3 \% \mathrm{Si}$ represented in the mode utilized in the VM based on the orientation measurement by ECP. The areas of the grains and grain orientations are taken into consideration in this representation.

The intensity distribution along $\zeta$ angle in the Box number 1 , 29 and 36 equivalent to the minor, medium and major texture components in this specimen, are shown in Figure 8. With the exception of Box number 36, agreement is not good. The numbers of grains measured by ECP were 116, 43 and 17 for Box 36, 29 and 1 , respectively. The low number of grains analyzed in Box numbers

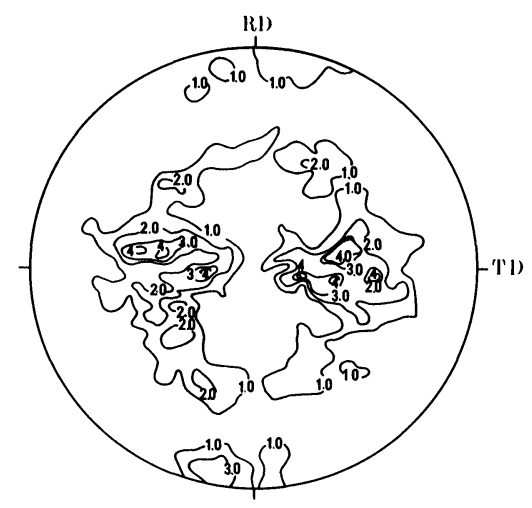

Figure 6 (100) pole figure (PFE) of $\mathrm{Fe}-3 \% \mathrm{Si}$ made by orientation measurements by ECP. The areas of the grains are taken into consideration in this figure. 


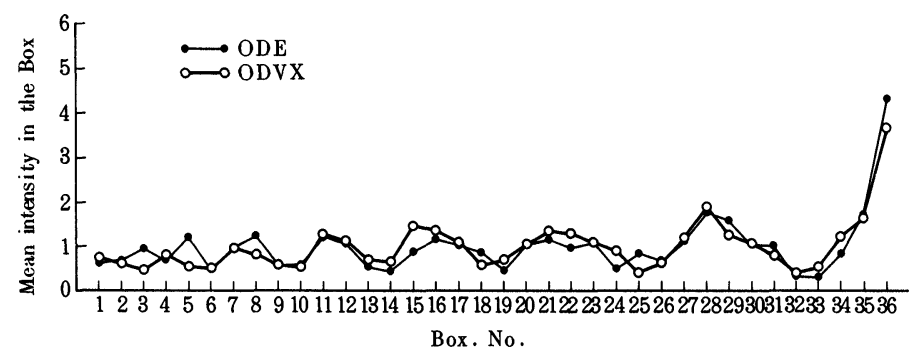

Figure 7 Comparison of the mean intensities in each Box between ODE and ODVX.

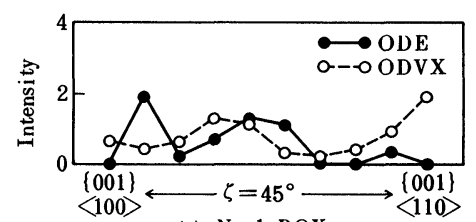

(a) No. 1 BOX
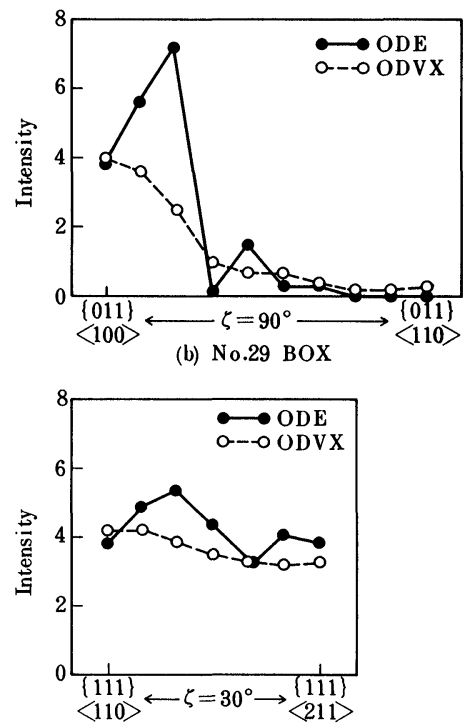

(c) No. 36 BOX

Figure 8 Comparison between ODE and ODVE of the intensities along the $\zeta$ angle in specific Boxes. 
Box No.

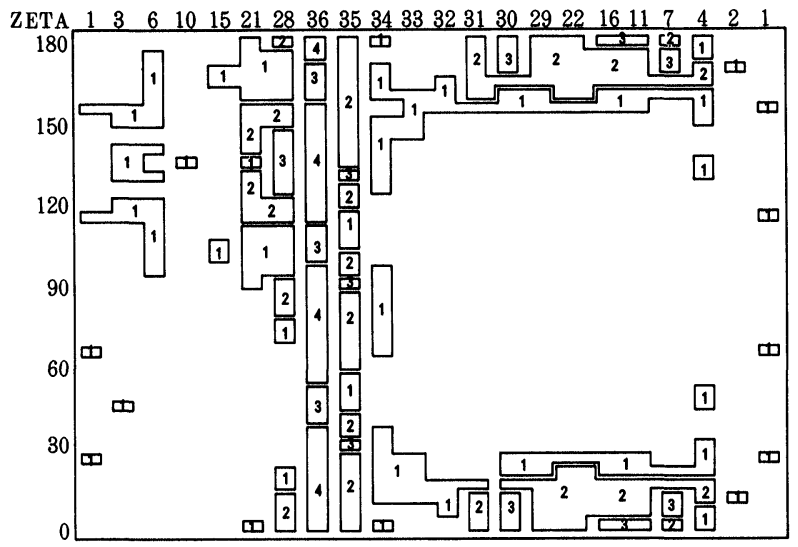

Figure 9 Three dimensional texture of $\mathrm{Fe}-3 \% \mathrm{Si}$ (ODVE) inverted from (100) pole figure shown in Figure 6 (PFE) by VM.

1 and 29 in ECP measurements is a possible cause of the larger discrepancy at these positions. This suggests that it is not appropriate to evaluate the accuracy of the minor texture component by VM as the numbers of grains measured by ECP is not sufficient for the statistical analysis. ODVE inverted by VM from the (100) pole figure in $\mathrm{Fe}-3 \% \mathrm{Si}$ (PFE shown in Figure 6 made by ECP) is shown in Figure 9. Figure 10 shows a comparison of the mean intensities of the individual Box numbers obtained by ODE and ODVE in $\mathrm{Fe}-3 \% \mathrm{Si}$. As seen previously in Figure 7, these data are also in very good agreement.

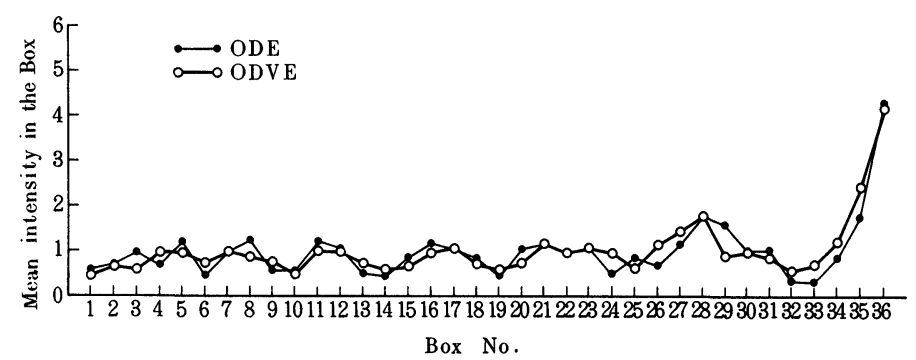

Figure 10 Comparison of the mean intensities in each Box between ODE and ODVE in $\mathrm{Fe}-3 \% \mathrm{Si}$. 


\section{COMPARISON OF ODE WITH ODVE IN FE-50\% Ni ALLOY}

Three specimens (1A, 5A and 1D) of primary and secondary recrystallized $\mathrm{Fe}-50 \% \mathrm{Ni}$ alloy were utilized in this investigation. The chemical compositions and the experimental procedure for the preparation of the specimens have been described previously (Harase et al., 1987). The number of grains analyzed by ECP were 206, 515 and 275 for specimen $1 \mathrm{D}, 5 \mathrm{~A}$, and $1 \mathrm{~A}$ respectively. The orientations of these grains are shown in Figures 11, 12 and 13 in the form of (100) pole figure and in standard stereographic triangles. In these figures each point represents one orientation and the grain area has not been considered. In contrast with $\mathrm{Fe}-3 \% \mathrm{Si}$ alloy used in the previous section, each specimen has several strong preferred orientations.

From the ND orientation, specimen 1D has preferred orientations of (8614), (12614), (4218) and (002). Specimen 1A has preferred orientations of (002), (12 614), (10616) and specimen 5A has preferred orientations of (002), (8614), (426) and (12614).

Pole figures which take account of the grain area of these three
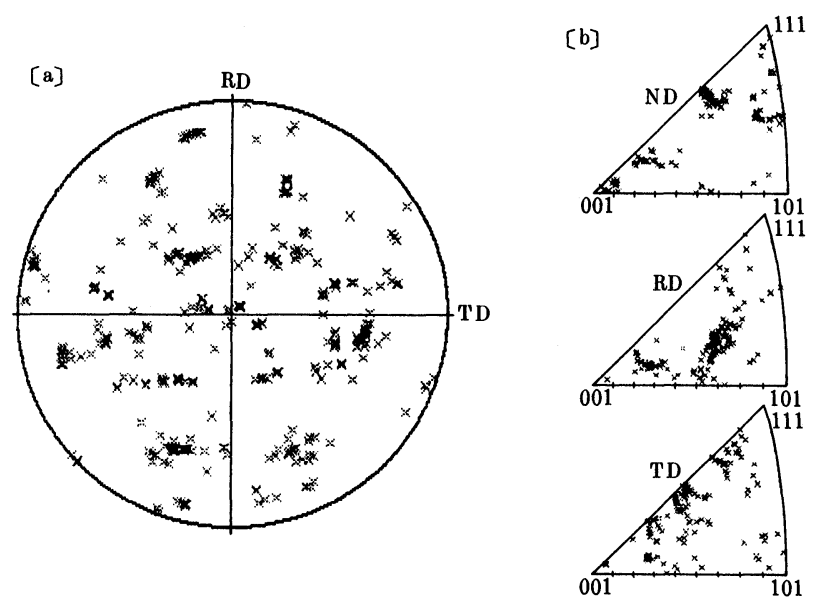

Figure 11 Orientation distribution of 206 grains analyzed by ECP in the specimen 1D. (a) (100) pole figure, (b) Standard stereographic triangle projections (inverse pole figures). The areas of grains are not taken into consideration in these figures. 
[b]
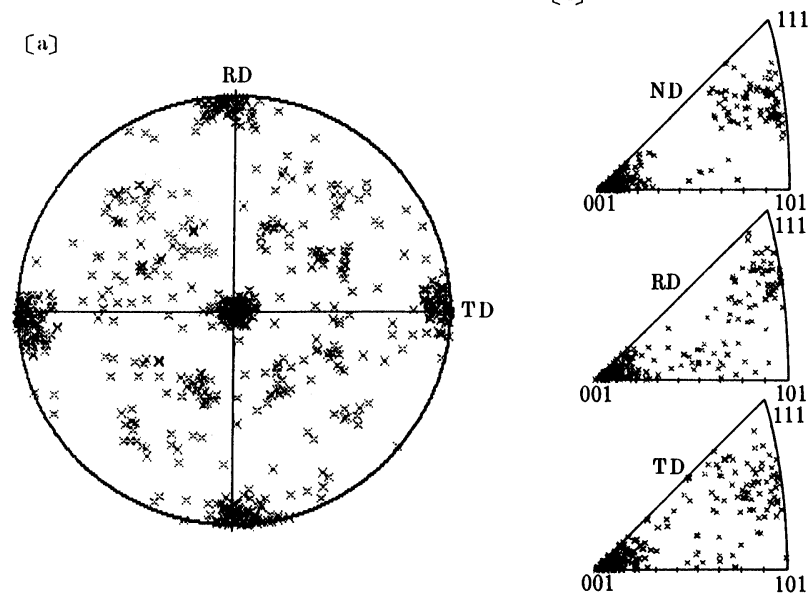

Figure 12 Orientation distribution of 275 grains measured by ECP in specimen $1 \mathrm{~A}$. (a) (100) pole figure, (b) Standard stereographic triangle projections (inverse pole figures). The areas of grains are not taken into consideration in these figures.

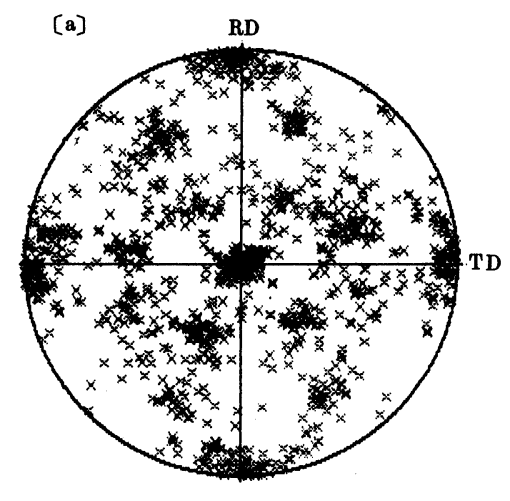

[b]

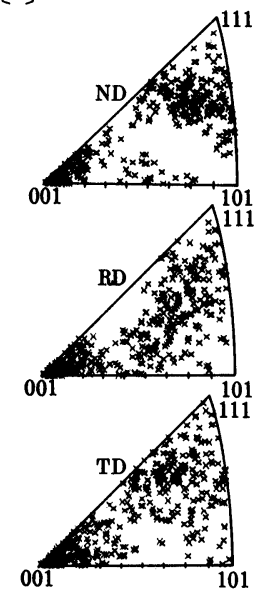

Figure 13 Orientation distribution of 515 grains measured by ECP in specimen 5A. (a) (100) pole figure, (b) Standard stereographic triangle projections (inverse pole figures). The areas of grains are not taken into consideration in these figures. 

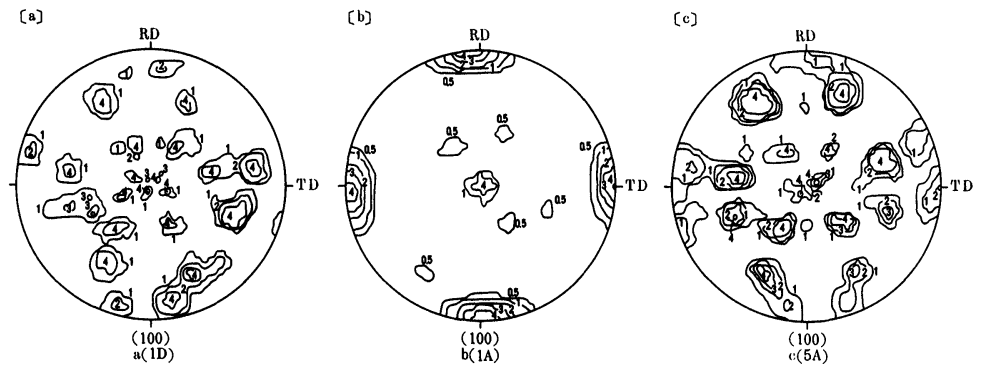

Figure 14 (100) pole figures (PTE) of three specimens shown in Figures 11, 12 and 13 respectively made by consideration of orientation and the area of individual grains. (a) 1D, (b) $1 \mathrm{~A}$, (c) $5 \mathrm{~A}$.

specimens (PFE) are shown in Figure 14. The mean intensities of the individual Box obtained by ODE and ODVE are shown in Figures 15, 16 and 17 for specimens $1 \mathrm{D}, 1 \mathrm{~A}$ and $5 \mathrm{~A}$ respectively.

Specimen 5A shows very good agreement and specimen $1 \mathrm{~A}$ shows a fairly good agreement, however, in specimen 1D the discrepancies at Box numbers 15, 20 and 21 are considerable. This is considered to be due to the sharpness of the texture in $1 \mathrm{D}$ for the following reasons.

- In the process of making PFE from the original orientation measurements by ECP, the intensity of an individual point in

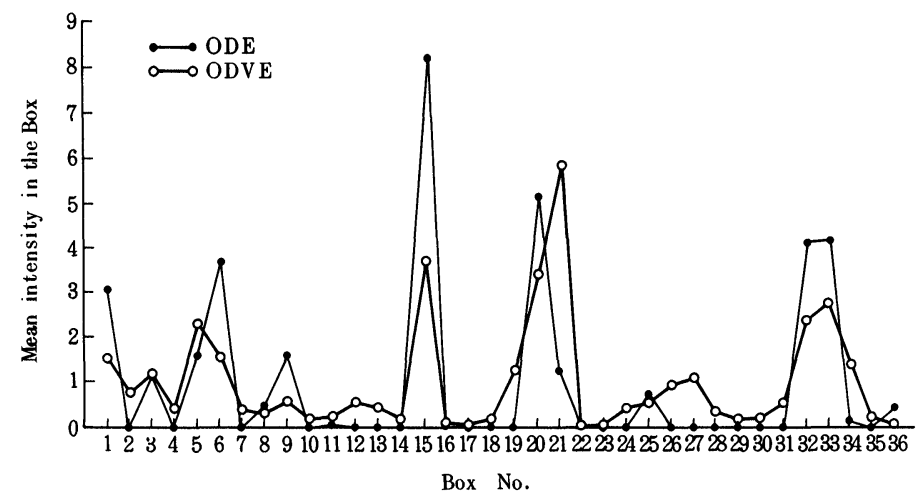

Figure 15 Comparison of mean intensities in each Box between ODE and ODVE (specimen 1D). 


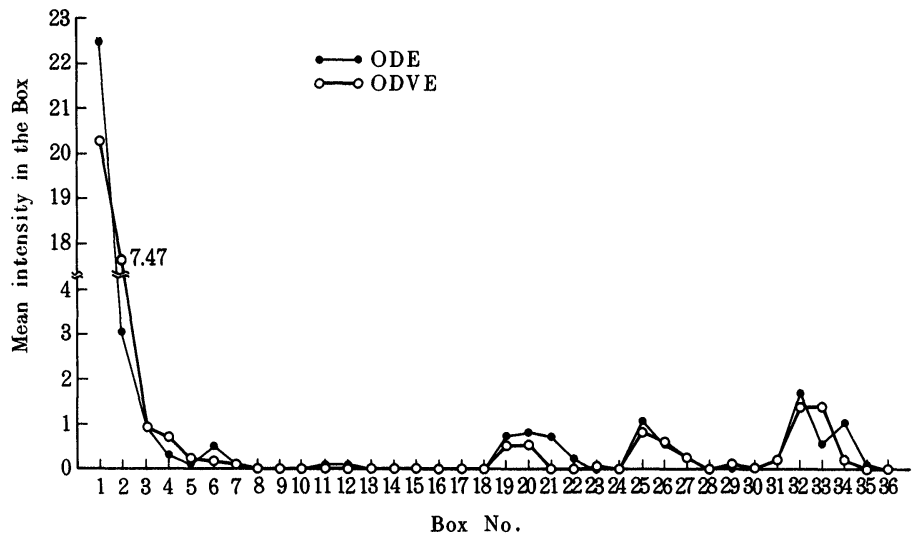

Figure 16 Comparison of mean intensities in each Box between ODE and ODVE (specimen 1A).

the pole figure is derived by averaging the intensities of several surrounding points.

- The intensity distribution of the original orientation measured by ECP is given in a Gaussian distribution in PFE. While the intensity of a point in ODE represents the original intensity measured by ECP, the intensity of the same point in ODVE is affected by the method used to generate the pole figure. This is either done by inversion using the vector method or by

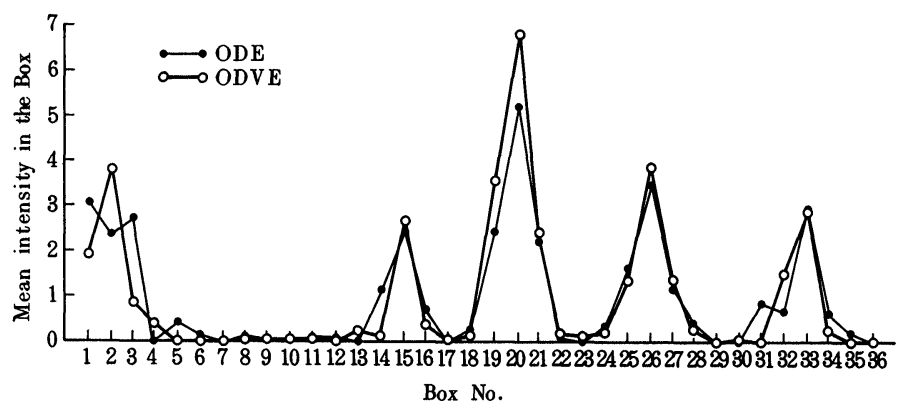

Figure 17 Comparison of mean intensities in each Box between ODE and ODVE (specimen 5A). 
considering the intensity of a point in ODVE to newly correspond to the average of the intensities of neighbouring points. Therefore the discrepancy between ODE and ODVE might increase when the texture is sharper. The total intensities of the three Box numbers $(15,20$ and 21) agree well having values of 14.8 and 12.9 for ODE and ODVE respectively.

\section{INVESTIGATIONS INTO THE ACCURACY OF THE DERIVED MINOR TEXTURE COMPONENTS BY VM}

It has been pointed out elsewhere (Matsuo et al., 1985) that the VM is not so reliable in the analysis of minor texture components, however, these conclusions were derived from an investigation utilizing model orientations. It has already been shown that one of the weak points of the VM is the large discrepancy appearing between the inverted texture by the VM and the given texture when model textures are utilized. In this connection, $\{110\}\langle\mathrm{uvw}\rangle$ orientations in a primary recrystallized $\mathrm{Fe}-3 \% \mathrm{Si}$ was used as the minor texture component. This is considered to be very important for the development of secondary recrystallization texture in this material. The average grain size of the specimen investigated was $7.5 \mu \mathrm{m}$. PFX and ODVX are shown in Figures $18 \& 19$, respectively. A surface area of $10 \mathrm{~mm} \times 10 \mathrm{~mm}$ was scanned by ECP mode perpendicular to the rolling direction (RD) at each $100 \mu \mathrm{m}$ interval. When the patterns indicating the $\{110\}\langle\mathrm{uvw}\rangle$ were detected during scanning, an ECP of the grain was taken and its grain size was measured by ECC mode. As the mean grain size of the specimen is $7.5 \mu \mathrm{m}$ the total area scanned by ECP is estimated about $8 \%$ of the area of $10 \mathrm{~mm} \times 10 \mathrm{~mm}$.

For the analysis of ECP, the rotation angle around ND, RD and TD axes is measured from the ECP directly instead of obtaining (HKL)[UVW] from the pattern because the rotation angle of $\{110\}\langle 001\rangle$ is important in this analysis. Counting the areas and numbers of grains belonging to the same ranges of rotation angle, the relative intensities were calculated and compared with ODVX inverted by the VM. The intensity distribution of $\{110\}\langle u v w\rangle$ orientation around ND axis corresponding to No. 29 Box of $\zeta=180^{\circ}$ to $90^{\circ}$ and $\zeta=0^{\circ}$ to $90^{\circ}$ in Figure 19 is shown in Figure 20. 


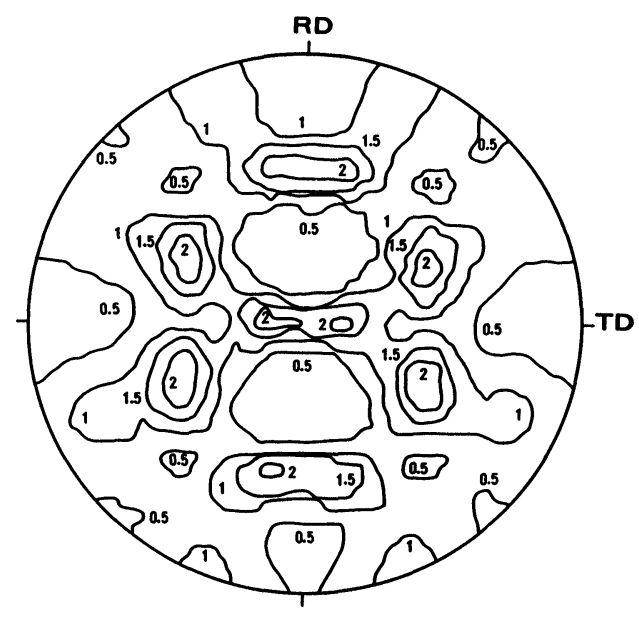

Figure 18 (100) pole figure (PFX) of recrystallized $\mathrm{Fe}-3 \% \mathrm{Si}$ for the investigation of the minor texture component.

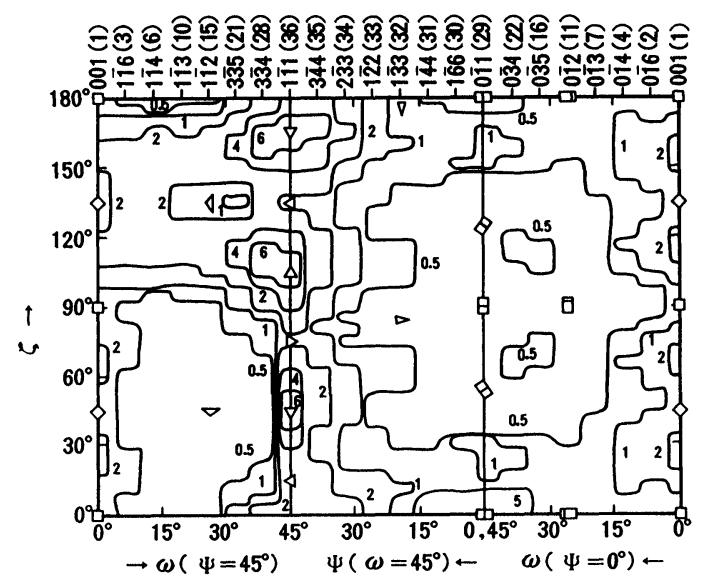

Figure 19 Inversion of (100) pole figure shown in Figure 18 by VM. 


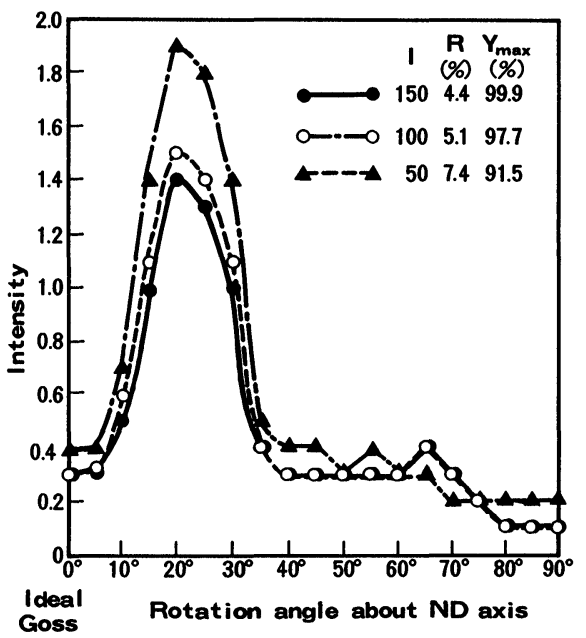

Figure 20 Intensity distribution of $\{110\}\langle\mathrm{uvw}\rangle$ along $\zeta$ angle analyzed by VM.

In the VM, each Box has an approximately $6^{\circ}$ spread in the $\psi$ and the $\omega$ angles. Therefore, the orientation rotated more than $6^{\circ}$ around the RD or the TD axis is classified either in Box No. 22 or Box No. 30, respectively. Among the analyzed results by ECP, orientations within $6^{\circ}$ from RD, TD axis were selected. The relative intensities around the ND axis are shown in Figure 21. The broken line in this figure is taken from Figure 20 for comparison. The shape of the relative intensities along $\zeta$ angle for both lines are in good agreement.

\section{CONCLUSIONS}

The orientation distributions (ODE) in recrystallized $\mathrm{Fe}-3 \% \mathrm{Si}$ and $\mathrm{Fe}-50 \% \mathrm{Ni}$ have been analyzed using both $\mathrm{ECP}$ and inversion results of pole figures by the VM. The results of this comparison are:

- ODE made from orientations and area measurements of 967 grains in $\mathrm{Fe}-3 \% \mathrm{Si}$ and ODVX inverted by $\mathrm{VM}$ from pole 


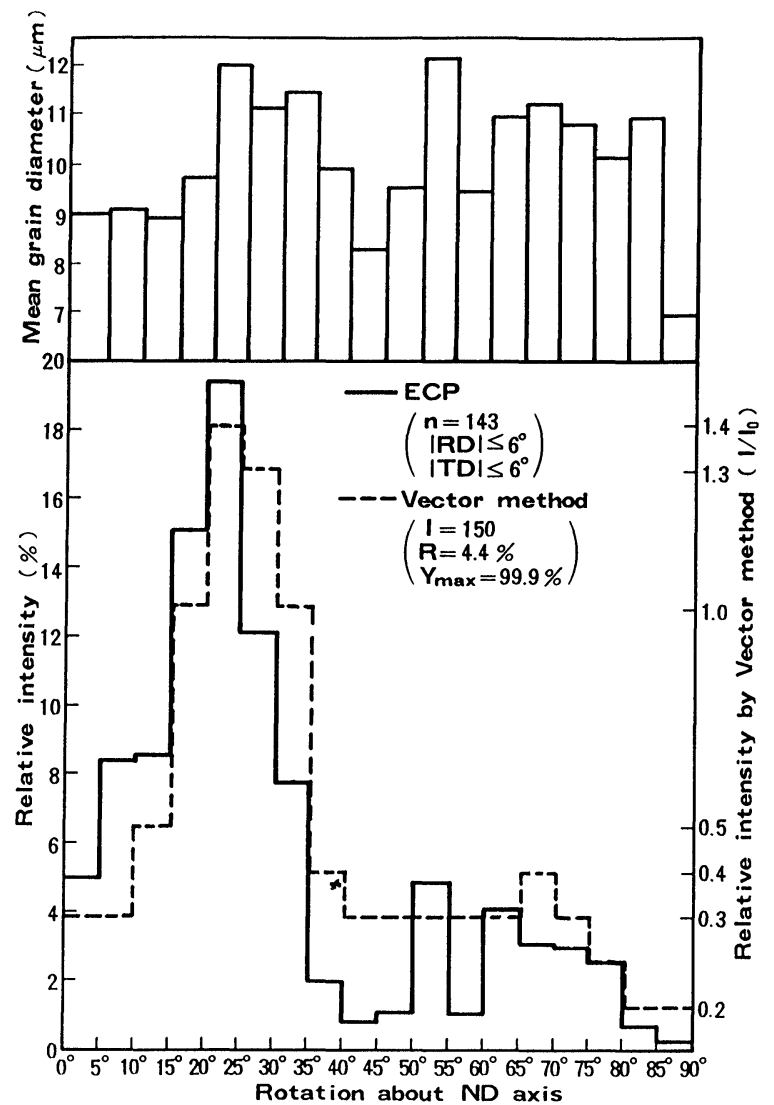

Figure 21 Comparison of the intensity distribution of $\{110\}\langle u v w\rangle$ along $\zeta$ angle obtained by VM and ECP.

figure (PFX) measured by X-ray were in good agreement with the mean intensities of each individual Box. In the No. 36 Box, which had a relatively large number of grains, the intensity distribution along $\zeta$ angle showed a relatively good agreement.

- In the recrystallized $\mathrm{Fe}-3 \% \mathrm{Si}, \mathrm{ODE}$ and ODVE were in good agreement for the mean intensities in the individual Boxes.

- In the recrystallized $\mathrm{Fe}-50 \% \mathrm{Ni}$, with weaker preferred texture (1A) good agreement between the different methods was 
obtained. For specimens with very strong preferred texture (1D, 5A), the agreement was not good, however, when the intensities from the several neighbouring Boxes were averaged, they showed good agreement.

- It was confirmed that quite a good agreement was obtained for the intensity distribution of the minor texture component of $\mathrm{Fe}-3 \%$ Si using ODVX and direct measurement by ECP.

- The mean intensities of the individual Box measured using ODE and ODVX were in good agreement, however, a comparison between ODE and ODVX in the intensity distribution along $\zeta$ angle could not be done because of the small number of grains investigated.

- For the minor texture component mentioned in (4), ODVX is considered to give correct values for both the mean intensities in the individual Boxes and for the intensity distribution along the $\zeta$ angle. Therefore it is concluded that the inversion of the pole figure (PFX) by the VM is accurate enough for most practical purposes provided that the starting input pole figure (PFX) is accurate. This is in agreement with previous work of Schaeben.

\section{Acknowledgment}

The authors would like to express sincere thanks for Dr. R. Davidson at Harwell in the UK for his critical reading and advice.

\section{References}

Esling, C., Bunge, H. J., Philippe, M. J. and Muller, J. (1987). Theoretical Methods of Texture Analysis. Ed.; H. J. Bunge. DGM. Oberusel.

Nagashima, S., Tanaka, K. and Nishikawa, S. (1981). Tetsu to Hagané, 67, S1198. Kitagawa, H., Morimoto, K. and Iwasaki, Y. (1982). Tetsu to Hagané, 68, S1351. Shimizu, R., Ohta, K. and Takahashi, N. (1983). Tetsu to Hagané, 69, S1280.

Pospiech, J., Romanska, M. and Lücke, J. (1984). Procc. 7th Inter. Conf. On Tex. Mat., 823.

Schaeben, H. (1984). Procc. 7th Inter. Conf. on Tex. Mat., 833.

Shimizu, R. and Ohta, K. (1984). Tetsu to Hagané, 70, S565.

Shimizu, R., Ohta, K., Harase, J. and Watanabe, T. (1984). Tetsu to Hagané, 70, S1322.

Matsuo, M., Kawasaki, K., and Sugai, T. (1985). Tetsu to Hagané, 71, S1351.

Harase, J., Shimizu, R. and Watanabe, T. (1987). Procc. 8th Inter. Conf. on Tex. Mat. 